\title{
Sauter-Schwinger pair creation dynamically assisted by a plane wave
}

\author{
Greger Torgrimsson, ${ }^{1,2,3}$ Christian Schneider, ${ }^{1}$ and Ralf Schützhold ${ }^{1}$ \\ ${ }^{1}$ Fakultät für Physik, Universität Duisburg-Essen, Lotharstraße 1, 47057 Duisburg, Germany \\ ${ }^{2}$ Theoretisch-Physikalisches Institut, Abbe Center of Photonics, Friedrich-Schiller-Universität Jena, \\ Max-Wien-Platz 1, 07743 Jena, Germany \\ ${ }^{3}$ Helmholtz Institute Jena, Fröbelstieg 3, 07743 Jena, Germany
}

(Received 11 January 2018; published 7 May 2018)

\begin{abstract}
We study electron-positron pair creation by a strong and constant electric field superimposed with a weaker transversal plane wave which is incident perpendicularly (or under some angle). Comparing the fully nonperturbative approach based on the world-line instanton method with a perturbative expansion into powers of the strength of the weaker plane wave, we find good agreement-provided that the latter is carried out to sufficiently high orders. As usual for the dynamically assisted Sauter-Schwinger effect, the additional plane wave induces an exponential enhancement of the pair-creation probability if the combined Keldysh parameter exceeds a certain threshold.
\end{abstract}

DOI: 10.1103/PhysRevD.97.096004

\section{INTRODUCTION}

As one of the most striking consequences of quantum field theory, extreme external conditions can tear apart quantum vacuum fluctuations and thereby create real particles. Already in 1939, Schrödinger predicted that the rapid expansion of the Universe could induce such a process [1]. As another example, the strong gravitational field around a black hole can tear apart quantum vacuum fluctuations leading to Hawking radiation, i.e., black hole evaporation [2,3]. In analogy to the gravitational force, a strong electric field can have a similar effect and create electron-positron pairs out of the quantum vacuum - the Sauter-Schwinger effect [4-7]. For a constant electric field $E$, the pair creation probability (per unit time and volume) scales as $(\hbar=c=1)$

$$
P_{e^{+} e^{-}} \sim \exp \left\{-\pi \frac{m^{2}}{q E}\right\}
$$

where $q$ and $m$ are the elementary charge and the mass of the positron/electron, respectively.

Unfortunately, this fundamental prediction of quantum field theory has not been directly verified experimentally yet because the required field strength is very large. This motivates the quest for ways to enhance the pair-creation probability or, equivalently, to lower the required field strength. One option is the dynamically assisted SauterSchwinger effect [8-14], where the pair-creation probability

Published by the American Physical Society under the terms of the Creative Commons Attribution 4.0 International license. Further distribution of this work must maintain attribution to the author(s) and the published article's title, journal citation, and DOI. Funded by SCOAP. is strongly enhanced by adding a weaker time-dependent field to the strong field $E$. So far, most studies of this enhancement mechanism have been devoted to purely time-dependent fields [15].

As a step towards a more realistic field configuration, we consider a propagating plane wave superimposed to the constant field $E$ in the following. Plane waves propagating parallel to the constant electric field were already considered in [17-20], for example. It was found that such transversal plane waves do not enhance the pair creation probability [18]. Further, for longitudinal parallel waves, $E_{z}(t+z)$, the pair creation probability is given by the locally constant field approximation [21-23], which implies that the enhancement is comparably small. Both results can be understood by considering a Lorentz boost along the direction of the strong field which leaves the strong field invariant but reduces the frequency of the plane wave, see also [24]. In the transversal case, the field strength of the plane wave is reduced as well while the longitudinal wave retains its field strength.

In contrast to the parallel scenarios discussed in [18-23], we consider the case of a transversal plane wave propagating perpendicular to the strong field $E$

$$
\boldsymbol{E}(t, x)=E \boldsymbol{e}_{z}+\varepsilon E \cos (\Omega[t-x]) \boldsymbol{e}_{z},
$$

corresponding to the vector potential (in temporal gauge) $A_{z}(t, x)=E t+\varepsilon E \sin (\Omega[t-x]) / \Omega$.

This scenario has several advantages: since such a transversal wave cannot create electron-positron pairs on its own (due to a similar Lorentz boost argument as above), pair creation can only occur in cooperation with the strong field $E$, which retains the nonperturbative character of this effect. Furthermore, the above profile (2) represents a vacuum solution to the Maxwell equations and could be 
a reasonable approximation for an experimental setup where $E$ represents the focus of an optical laser while the plane wave is generated by an X-ray free-electron laser (XFEL). Finally, we found that this scenario (2) yields the maximum enhancement of the pair creation probability. Other profiles, polarizations and propagation directions will be discussed below in Sec. III and Appendix A. Note that this profile (2) was already considered in [25] using first-order perturbation theory in $\varepsilon$, whereas we are going to consider higher orders as well as a fully nonperturbative approach.

\section{PERTURBATIVE APPROACH}

At first, we employ a perturbative expansion of the total pair creation probability $P_{e^{+} e^{-}}$in powers of the relative strength $\varepsilon$ of the plane wave, which is supposed to be small $\varepsilon \ll 1$

$$
P_{e^{+} e^{-}}=\sum_{N=0}^{\infty} \varepsilon^{N} P_{N}
$$

where the contributions $P_{N}$ can be derived via the world-line formalism, for an introduction see [26,27] and references therein. The zeroth order $N=0$ reproduces the original Sauter-Schwinger effect in Eq. (1), and odd orders vanish in this situation (but not always [28]).

The lowest-order term $N=2$ corresponding to the onephoton contribution has already been calculated in [25]. Deriving the exponential dependence for the higher-order terms, it turns out that the exponent for two photons ( $N=4)$ with frequency $\Omega$ is the same as that for a single photon $(N=2)$ with twice the frequency $2 \Omega$, and so on for more photons (see Appendix A).

Consequently, we find

$$
P_{N} \sim \exp \left\{-\frac{2 m_{\perp}^{2}}{q E}\left(\arccos \Sigma-\Sigma \sqrt{1-\Sigma^{2}}\right)\right\},
$$

where the function of $\Sigma$ in the exponent is already known from [14]. The effective mass $m_{\perp}=\sqrt{m^{2}+(N \Omega / 4)^{2}}$ reflects momentum conservation in $x$-direction, where the momentum $N \Omega / 2$ of the $N / 2$ photons has to be transferred to the electron-positron pair. As a result, the effective mass $m_{\perp}$ is higher than the original mass $m$, and hence the pair creation probability is lower than in the case of a purely time-dependent field. Finally, $\Sigma$ describes the relative contribution of the energy of the $N / 2$ photons in comparison to the effective mass gap $2 m_{\perp}$

$$
\Sigma=\frac{N \Omega / 2}{2 m_{\perp}}=\frac{N \Omega}{4 \sqrt{m^{2}+(N \Omega / 4)^{2}}} .
$$

In the limit of $\Omega \downarrow 0$, i.e., $\Sigma \downarrow 0$, where $m_{\perp} \downarrow m$, we recover Eq. (1), as expected.

\section{A. Dominant order}

Inspecting the terms in the sum (3) we find that the prefactors $\varepsilon^{N}$ decrease as $N$ increases (due to $\varepsilon \ll 1$ ) while the exponentials in $P_{N}$ grow according to Eq. (4). As a result, there could be a dominant order $N_{*}$ which yields the maximum contribution to the sum (3). In order to study this question, we approximately treat $N$ as a continuous variable and apply the saddle point method to the term $\varepsilon^{N} P_{N}$, i.e.,

$$
\frac{\mathrm{d}}{\mathrm{d} N}\left\{-N|\ln \varepsilon|-\frac{2 m_{\perp}^{2}}{q E}\left(\arccos \Sigma-\Sigma \sqrt{1-\Sigma^{2}}\right)\right\}=0 .
$$

This yields the dominant order $N_{*}$ as solution of the transcendental equation

$$
1-\frac{N_{*} \Omega}{4 m} \arctan \frac{4 m}{N_{*} \Omega}=\frac{q E}{m \Omega}|\ln \varepsilon|=\frac{\gamma_{\text {crit }}}{\gamma},
$$

where we have introduced the (combined) Keldysh parameter $\gamma=m \Omega /(q E)$ and its threshold $\gamma_{\text {crit }}=|\ln \varepsilon|$. We only obtain real solutions $N_{*}$ if the right-hand side is less than unity, i.e., if $\gamma$ exceeds the threshold $\gamma_{\text {crit }}$. At the threshold, $\gamma=\gamma_{\text {crit }}$, we find $N_{*}=0$ which implies the original Schwinger result (1). For $\gamma>\gamma_{\text {crit }}$ and $\Omega \ll m$, however, the dominant order $N_{*}$ can be quite large (which justifies the continuum approximation). For example, for $\gamma=3 \gamma_{\text {crit }}$, the dominant order $N_{*}$ scales as $N_{*} \sim m^{2} /(q E|\ln \varepsilon|)$ which can be a large number for electric fields $E$ well below the Schwinger limit $E_{S}=m^{2} / q$.

In the limit $\gamma / \gamma_{\text {crit }} \gg 1$, we may approximate the solution of the transcendental equation (7) via

$$
N_{*}\left(\gamma \gg \gamma_{\text {crit }}\right) \approx \frac{4 m}{\Omega} \sqrt{\frac{\gamma}{3 \gamma_{\text {crit }}}},
$$

which will also be a large number unless the frequency $\Omega$ far exceeds the electron mass $m$. Inserting this approximate solution for the dominant order $N_{*}$ back into the exponent (4), we find

$$
P_{e^{+} e^{-}} \sim \exp \left\{-8 \frac{m^{2}}{q E} \sqrt{\frac{\gamma_{\text {crit }}}{3 \gamma}}\right\} .
$$

In contrast to the dynamically assisted Sauter-Schwinger effect with a purely time-dependent field, we see that the exponent still crucially depends on the strong field $E$, which demonstrates the nonperturbative character of this effect even for $\gamma / \gamma_{\text {crit }} \gg 1$. As mentioned in the Introduction, this is a consequence of the fact that a plane wave alone cannot create electron-positron pairs out of the vacuum.

\section{B. Improved approximation}

In the following, we try to improve the accuracy of the approximation outlined in the previous section. The above estimate of the leading order $N_{*}$ was based on the competition between the factor $\varepsilon^{N}$ and the exponent (4). However, the prefactor in front of this exponent will also depend on $N$ and thereby slightly modify the scaling 
with $N$. Thus, in order to improve our approximation, we make an educated guess for the scaling of that prefactor with $N$. Obviously, each additional power of $\varepsilon$ must be accompanied by a factor of $q E$ since this governs the coupling to the fermionic field. Recalling the structure of the QED interaction (vertex) term $\bar{\psi} q A_{\mu} \gamma^{\mu} \psi$, it seems quite reasonable to suppose a scaling with $\varepsilon q E / \Omega$ since $A_{\mu} \propto E / \Omega$. Finally, in view of dimensionality arguments, we arrive at the following rough estimate for the scaling of the prefactor:

$$
\begin{aligned}
\varepsilon^{N} P_{N} \sim & \left(\varepsilon \frac{q E}{m \Omega} \times \text { const }\right)^{N} \\
& \times \exp \left\{-\frac{2 m_{\perp}^{2}}{q E}\left(\arccos \Sigma-\Sigma \sqrt{1-\Sigma^{2}}\right)\right\},
\end{aligned}
$$

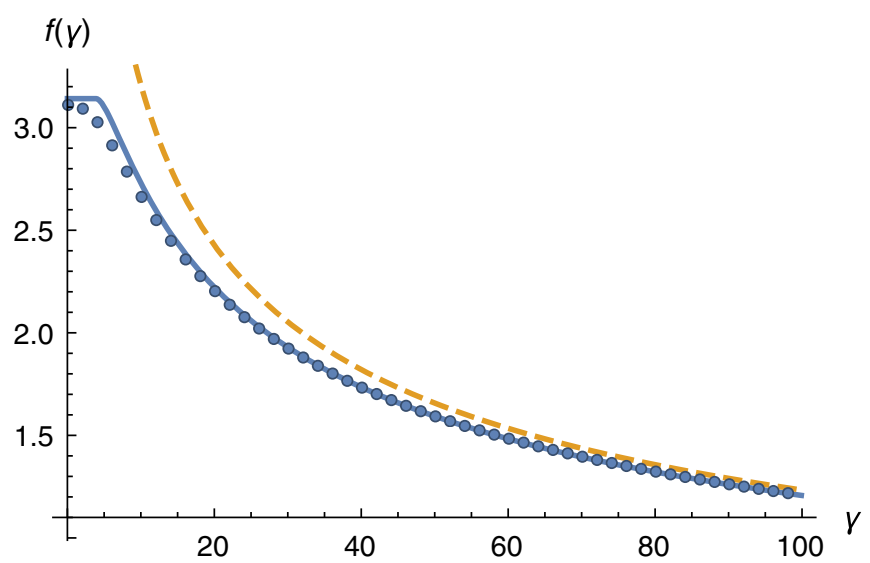

$f(\gamma)$

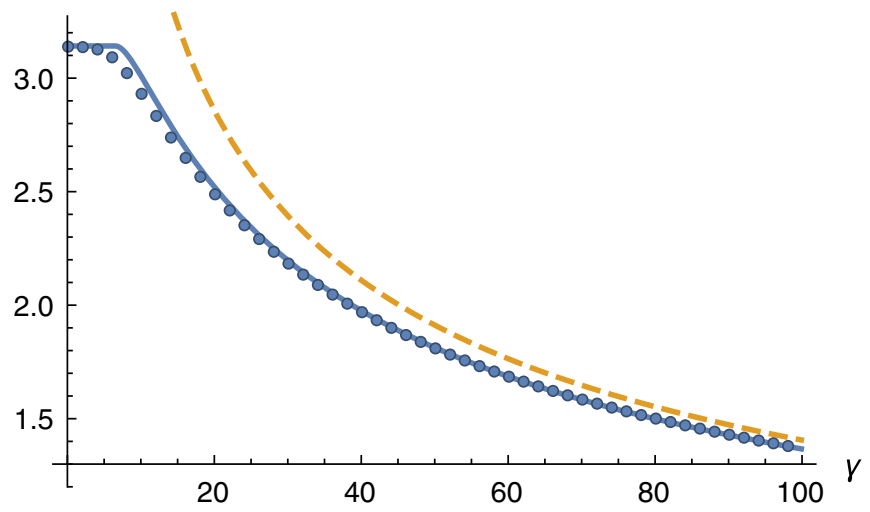

FIG. 1. Plot of the exponent of the pair-creation probability $P_{e^{+} e^{-}}$ as a function of $\gamma$ for perpendicular incidence and parallel polarization (2) with $\varepsilon=10^{-2}$ (top) and $\varepsilon=10^{-3}$ (bottom). The exponent has been multiplied by $q E / m^{2}$, i.e., the plot shows $f(\gamma)$ such that $P_{e^{+} e^{-}} \sim \exp \left\{-f(\gamma) m^{2} /[q E]\right\}$. The circles represent the numerical world-line instanton results from Sec. IV, and the dashed curve corresponds to the large- $\gamma$ approximation in (9). The solid curve shows the result of our improved analytical approximation obtained by inserting the dominant order $N_{*}$ from Eq. (11) into Eq. (4). The constant factor in Eq. (11) has been chosen in order to match the world-line instanton results, which gives a factor of 8 for $\varepsilon=10^{-2}$ (top) and a factor of 9.5 for $\varepsilon=10^{-3}$ (bottom). With these values, we observe good agreement between our improved analytical approximation and the numerical world-line instanton results.
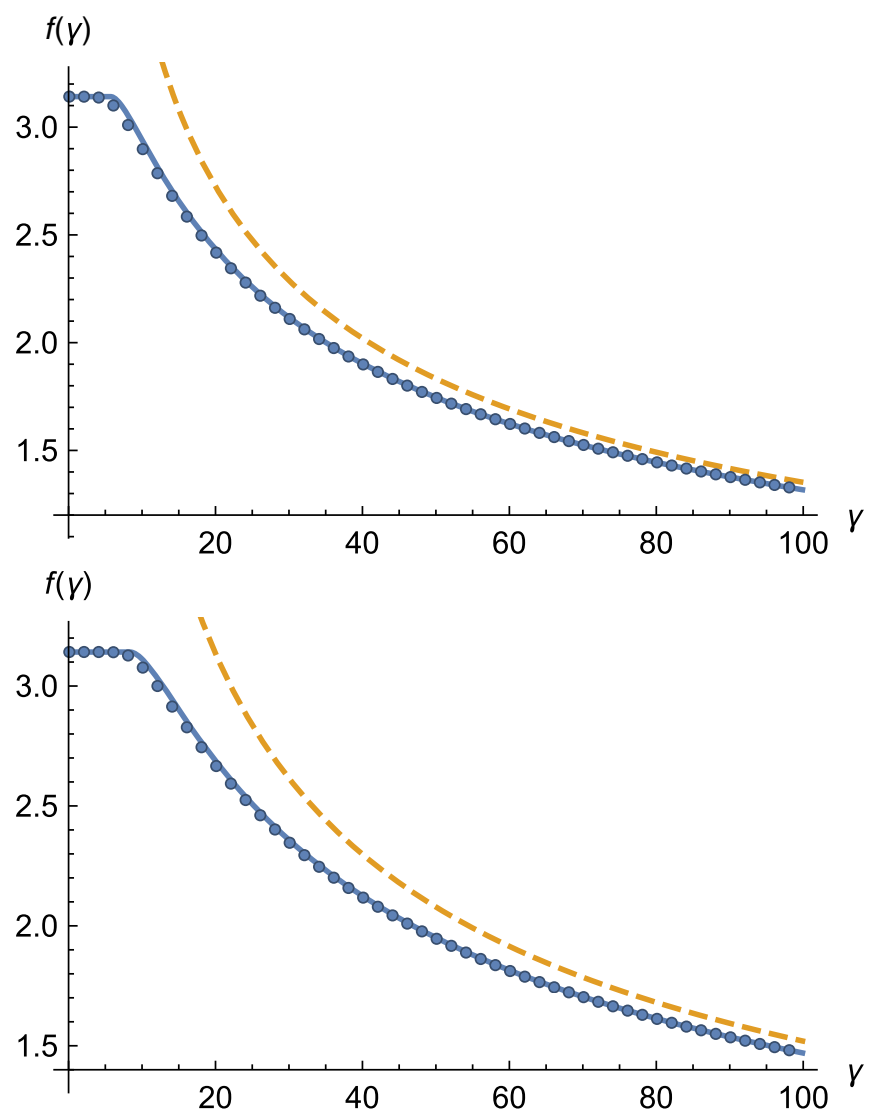

FIG. 2. Same as Fig. 1, but for perpendicular polarization $\boldsymbol{e}_{P}^{\perp}$ as discussed in Sec. III. Again, the constant factor in Eq. (11) is obtained by fitting and gives 1.9 for $\varepsilon=10^{-2}$ (top) and 2 for $\varepsilon=10^{-3}$ (bottom). We observe that perpendicular polarization yields a lower pair-creation probability $P_{e^{+} e^{-}}$.

where const is a (so far undetermined) constant—or, more precisely, a factor which should only weakly depend on the involved parameters.

The saddle point of the above expression gives a slightly shifted dominant order $N_{*}$ as a solution of the transcendental equation with a modified right-hand side

$1-\frac{N_{*} \Omega}{4 m} \arctan \frac{4 m}{N_{*} \Omega}=\frac{q E}{m \Omega} \mid \ln \left(\varepsilon \frac{q E}{m \Omega} \times\right.$ const $) \mid$.

Comparison with fully nonperturbative results obtained with the world-line instanton method as described in Sec. IV shows that this modification is indeed an improvement of our approximation and leads to good agreement, see Figs. 1 and 2.

\section{OTHER DIRECTIONS}

So far, we have considered the case of perpendicular incidence and a plane wave with the electric field component parallel to the strong field (2), which yields the maximum enhancement. Now let us briefly discuss more general angles 


$$
\boldsymbol{E}(t, \boldsymbol{r})=E \boldsymbol{e}_{z}+\varepsilon E \cos (\Omega t-\boldsymbol{K} \cdot \boldsymbol{r}) \boldsymbol{e}_{P},
$$

where the corresponding vector potential reads $A_{0}=0$ and $\boldsymbol{A}(t, \boldsymbol{r})=E t \boldsymbol{e}_{z}+\varepsilon E \boldsymbol{e}_{P} \sin (\Omega t-\boldsymbol{K} \cdot \boldsymbol{r}) / \Omega$. Without loss of generality, we may set $\boldsymbol{K}=K_{\|} \boldsymbol{e}_{z}+K_{\perp} \boldsymbol{e}_{x}$ where $K_{\perp} \geq 0$. The polarization vector $\boldsymbol{e}_{P}$ obeys $\boldsymbol{K} \cdot \boldsymbol{e}_{P}=0$, for example $\boldsymbol{e}_{P}^{\perp}=\boldsymbol{e}_{y}$.

Inserting this more general field profile (12), we obtain formally the same results as in the previous section with $\Omega$ being replaced by $K_{\perp}$. Since the enhancement of the paircreation probability is monotonic in $\Omega$ (i.e., now $K_{\perp}$ ), we see that the perpendicular case with $K_{\|}=0$ and $K_{\perp}=\Omega$ is indeed optimal, see also [25].

Note that, while the exponents do not depend on the polarization vector $\boldsymbol{e}_{P}$, the prefactors do depend on $\boldsymbol{e}_{P}$, cf. [25], which can also generate a slight polarization dependence of the dominant order $N_{*}$ via the constant factor in (11). This is consistent with the results of the next section, which show that the world-line instantons, and their actions do also depend on the polarization vector $\boldsymbol{e}_{P}$.

\section{WORLD-LINE INSTANTONS}

We can express the probability for pair creation using the vacuum persistence amplitude $\left\langle 0_{\text {out }} \mid 0_{\text {in }}\right\rangle$ and thus the effective action $\Gamma$ with $\left\langle 0_{\text {out }} \mid 0_{\text {in }}\right\rangle=e^{i \Gamma}$

$$
P_{e^{+} e^{-}}=1-\left|\left\langle 0_{\text {out }} \mid 0_{\text {in }}\right\rangle\right|^{2}=1-e^{-2 \Im \Gamma} \approx 2 \Im \Gamma .
$$

The world-line instanton method is a semiclassical evaluation of the world-line path integral for the Euclidean effective action [29-31] (Euclidean because we replace time by imaginary time $x_{4}=i t$, it is related to the Minkowskian quantity by $\Gamma=i \Gamma^{\mathrm{E}}[31]$ ),

$$
\begin{aligned}
\Gamma^{\mathrm{E}}= & \int_{0}^{\infty} \frac{\mathrm{d} T}{T} e^{-\frac{m^{2}}{2} T} \int \mathcal{D} x(\tau) \Phi[x] \\
& \times \exp \left[-\int_{0}^{T} \mathrm{~d} \tau\left(\frac{\dot{x}^{2}}{2}+i q A^{\mathrm{E}} \cdot \dot{x}\right)\right],
\end{aligned}
$$

where the paths $x(\tau)$ are (in general four-dimensional) closed trajectories parametrized by the proper time $\tau$ with a period $T, A_{\mu}^{\mathrm{E}}(x(\tau))$ is the Euclidean four-potential evaluated on the trajectory and

$$
\Phi[x]=\frac{1}{2} \operatorname{tr}_{\Gamma} \mathcal{P} e^{\frac{1}{4} \int_{0}^{T} \mathrm{~d} \tau \sigma_{\mu \nu} i q F_{\mu \nu}^{\mathrm{E}}(x(\tau))}
$$

is the spin factor with $\sigma_{\mu \nu}=\frac{1}{2}\left[\gamma_{\mu}, \gamma_{\nu}\right], \operatorname{tr}_{\Gamma}$ denoting the trace over spinor indices and $\mathcal{P} e^{\cdots}$ the path ordered exponential. In this section we will only work with Euclidean quantities, so we omit the superscript ${ }^{\mathrm{E}}$ for brevity.

A saddle point evaluation for both the $T$ - and the path integral consists of finding periodic solutions that satisfy the Euler-Lagrange equations corresponding to the exponent in (14), that is, $m \ddot{x}_{\mu}(\tau)=a q i F_{\mu \nu}(x(\tau)) \dot{x}_{\nu}(\tau), \quad a^{2}=\dot{x}^{2}=$ const,

and evaluate their action which gives the exponential dependence of the pair production rate. The subleading prefactor is given by quadratic fluctuations around such solutions. For simple fields, $i F_{\mu \nu}$ is real (the Euclidean potential is purely imaginary) and (16) can often be restricted to a 2D-plane, sometimes even solved analytically [30,31]. In slightly more complicated fields, solutions can be found using a shooting method, numerically integrating (16) using initial conditions that are varied until the periodicity condition is met [32]. This is not feasible here, as the instantons are genuinely three-dimensional in the parallel polarization case and four-dimensional for other polarizations. Furthermore, they are not even purely real: we choose the Euclidean four potential (for parallel polarization)

$$
i A_{4}=E x_{3}, \quad i A_{3}=i \frac{\varepsilon E}{\Omega} \sin \left(\Omega\left(x_{1}-i x_{4}\right)\right) .
$$

Without the $x_{1}$-dependence, this would give real instanton equations (as considered in [12,33]), but in this case real and imaginary parts mix, see Fig. 3.

To robustly find instantons and evaluate both the exponent and the prefactor in such fields, we employ a method that will be discussed in detail elsewhere [34] and only provide the basic ideas here. Instead of a numerical integration of (16) (arising in a saddle point approximation

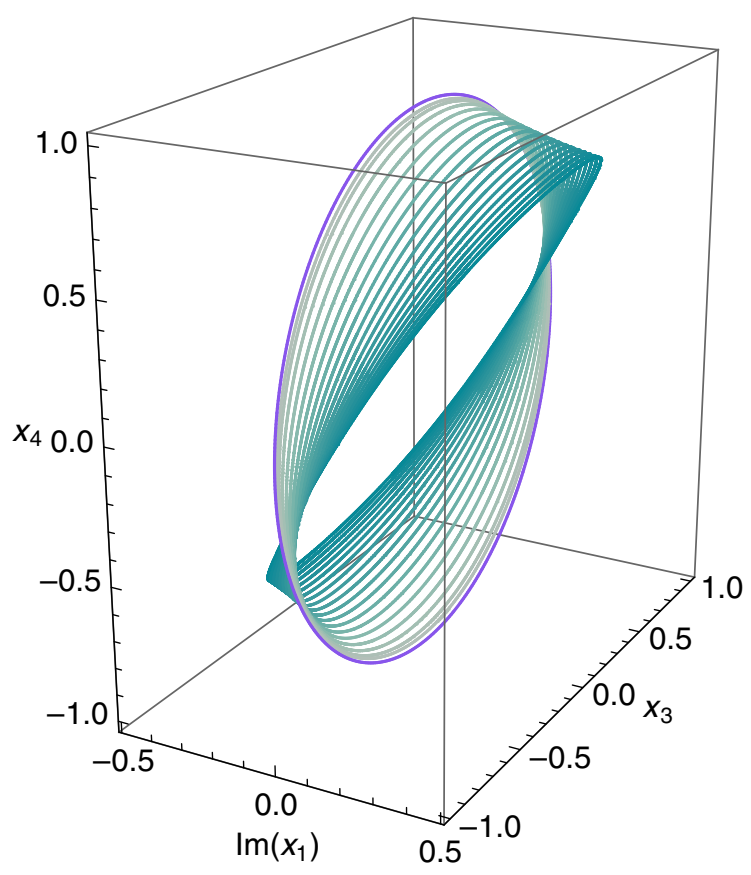

FIG. 3. Worldline instantons (in units of $m / q E$ ) for the case of parallel polarization with $\varepsilon=10^{-2}$ and $\gamma=m \Omega / q E$ ranging from 0 to 15. The purple circle in the $x_{1}=0$-plane is the circular instanton in the limit $\gamma \rightarrow 0$. For increasing values of $\gamma$ the instantons shrink (predominantly in the $x_{3}$-direction) and rotate in the $\Im\left(x_{1}\right)-x_{4}$-plane. 
of the continuous path integral), we discretize the paths in (14) into $N$ points from the beginning and perform the saddle point method on the resulting $N \times d$-dimensional integral, where $d$ is the number of space-time dimensions.

The equations of motion (16) are then replaced by a system of $N \times d$ nonlinear equations in $N \times d$ unknowns, which can be solved efficiently using a Newton-Raphson scheme, provided we choose a sufficiently close initial guess. In our case, the $\gamma \rightarrow 0$ limit corresponds to a static, homogeneous field so we can start with the known circular instanton, solve for the instanton at a small, finite $\gamma$ and use that as initial guess for the next value. This process is called natural parameter continuation [35] and is essentially what was used in [36]. We can improve on this using a more sophisticated predictorcorrector algorithm, also detailed in [34].

Note that the question of whether instanton solutions exist for a given (and possibly complicated) field configuration can be nontrivial. Here, we address this issue by starting with a known instanton solution and then changing the parameters gradually. Then, via analyticity arguments, one would expect continuously modified instanton solutions to exist—unless one hits a critical point in parameter space where the instanton develops a singularity. (For example, if the electrostatic potential difference drops below the mass gap, the instanton grows infinitely large and pair creation stops.) In the present case, one does not encounter such difficulties, and we have found instanton solutions for all considered parameters.

Having found instantons for different values of the Keldysh parameter $\gamma$ we can evaluate the instanton action to obtain the leading exponential contribution to the effective action. We can also evaluate the prefactor, which is just given by the inverse square root of the Hessian matrix $\mathbf{H}$. We do need to regularize zero modes arising in the integral, due to reparametrization and translational invariance. We deal with them using the Faddeev-Popov method [37,38], exponentiating the Dirac delta function, which modifies the Hessian to remove zero eigenvalues (details, again, in [34]). The final semiclassical result is then

$$
\frac{\Gamma}{V_{N_{0}} m^{N_{0}}} \approx\left(\frac{E}{E_{\mathrm{S}}}\right)^{\frac{N_{0}}{2}} \sqrt{\frac{2 \pi}{a^{\mathrm{cl}}}}\left(\frac{N}{a^{\mathrm{cl}}}\right)^{\frac{N d}{2}} \frac{\Phi\left[x^{\mathrm{cl}}\right] e^{-\frac{E_{\mathrm{S}}}{E} \mathcal{A}\left[x^{\mathrm{cl}}\right]}}{\sqrt{\operatorname{det} \mathbf{H}\left[x^{\mathrm{cl}}\right]}},
$$

where $N_{0}$ is the number of invariant directions, $V_{N_{0}}$ the corresponding volume factor, $x^{\mathrm{cl}}$ the discrete instanton (the collection of points) and $a^{\mathrm{cl}}$ its velocity. The trajectory and $a$ are made dimensionless by rescaling with $m / q E$.

The expression (18) has the advantage that it is applicable for every electromagnetic background field, yielding the correct prefactor including spin effects without having to compare limiting cases to determine normalization factors. Also the instantons are independent of the field strength, so as soon as they are computed, we can evaluate (18) for many different values of $E / E_{\mathrm{S}}$.

Figure 4 shows evaluations of (18) for both parallel and perpendicular polarization, in the perpendicular case with

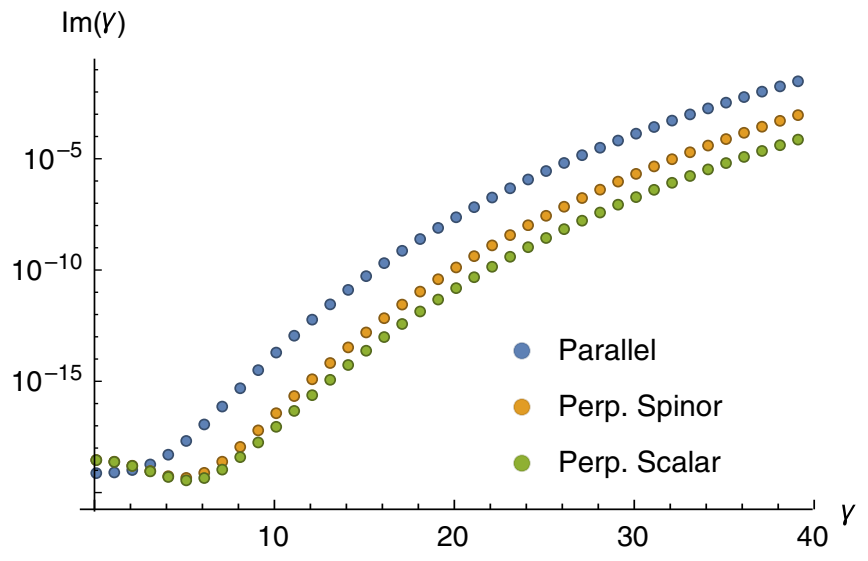

FIG. 4. Pair production probabilities for different values of the combined Keldysh parameter $\gamma$, strong field strength $E=E_{\mathrm{S}} / 30$ (corresponding to a laser intensity of $I \approx 5 \times 10^{26} \mathrm{~W} / \mathrm{cm}^{2}$ ) and weak field $\varepsilon=10^{-2}$. We have assumed a spacetime volume of $1 \mu \mathrm{m}^{4}$. As shown before, the case of parallel polarization yields stronger enhancement. Further, the spin factor does not contribute in the parallel case, while it enhances pair production in the perpendicular case. The values $\Omega=500 \mathrm{keV}$ or $\Omega=1 \mathrm{MeV}$ considered in [25], for example, would correspond to $\gamma=30$ or $\gamma=60$, respectively, and hence result in a drastic enhancement. Unfortunately, however, these values are probably outside the range of near future XFELs. Lower values such as $25 \mathrm{keV}$ [39] correspond to $\gamma=3 / 2$ (when $E=E_{\mathrm{S}} / 30$ ) and are thus not sufficient for an exponential enhancement. On the other hand, for lower field strengths such as $E=E_{\mathrm{S}} / 100$, the same frequency of $25 \mathrm{keV}$ would correspond to $\gamma=5$ where we start to see exponential enhancement. However, the total probabilities for $E=E_{\mathrm{S}} / 100$ are much lower and thus very hard to detect.

and without including the spin factor. While there is no spin dependence in the parallel case, the spin factor further enhances pair production for perpendicular polarization. The nonmonotonic behavior for small $\gamma$ in the perpendicular case is probably a sign that the saddle point approximation breaks down, as the instanton is not confined strongly enough in the $x_{4}$-direction. This has already been verified in the purely time dependent case in [33], where a comparison with the (numerical) solution of the Riccati equation could be made. For the volume factor we have assumed that the strong field ranges over a fourvolume of $1 \mu \mathrm{m}^{4}$, which is completely covered by the plane wave. It does not matter how much further the plane wave actually extends, as it cannot produce any pairs without the strong field. We thus hold e.g. $x_{1}(0), x_{2}(0)$ and $x_{3}(0)$ or their center of mass fixed, giving the three-volume $V_{3}$ in (18) and sum over the instantons located at each maximum of the wave giving a factor of $N_{\text {inst }}=T \Omega / 2 \pi$.

\section{CONCLUSIONS}

As an example for the dynamically assisted SauterSchwinger effect, we studied electron-positron pair creation due to a strong and constant electric field $E$ superimposed 
by a weaker transversal plane wave with a frequency $\Omega$ and field strength $\varepsilon E$. In analogy to other examples, we found an exponential enhancement of the pair-creation probability if the combined Keldysh parameter $\gamma=m \Omega /(q E)$ exceeds a threshold value $\gamma_{\text {crit }}$ which scales in the same way $\gamma_{\text {crit }} \sim$ $|\ln \varepsilon|$ as for a purely time-dependent sinusoidal field. However, the exponential enhancement above the threshold $\gamma>\gamma_{\text {crit }}$ is reduced in comparison to a purely timedependent sinusoidal field due to the effective mass $m_{\perp} \geq m$ stemming from momentum conservation.

In order to treat this genuinely space-time dependent field, we employed an analytical approach based on a perturbative expansion (3) into powers $\varepsilon^{N}$ of the weaker plane wave (while taking into account the strong field $E$ nonperturbatively). The exponential dependence (4) of the $N$ th order allows us to infer via (7) a dominant order $N_{*}$, which yields the strongest contribution and can be quite large $N_{*} \gg 1$, see also Appendix B. For large $\gamma$, we obtain the asymptotic expressions (8) and (9). Finally, we compare these analytical results to a fully nonperturbative numerical method based on the world-line instanton technique and find good agreement, e.g., regarding the dominant order $N_{*}$ (see Appendix B) and the pair-creation exponent in Figs. 1 and 2, especially after inserting an improved approximation (10) based on an ansatz for the subleading scaling of the prefactor (including one fitting parameter, fixed by the numerical results).

Note that the world-line instanton action $\mathcal{A}_{\text {inst }}$, which yields the exponent in the pair-creation probability $P_{e^{+} e^{-}} \sim \exp \left\{-\mathcal{A}_{\text {inst }}\right\}$, depends on the polarization and propagation direction. We find that perpendicular incidence with parallel polarization (2) yields maximum enhancement, see Fig. 4 and [25]. Furthermore, the prefactor in front of the exponential contains the volume scaling. Since the plane wave is supposed to be filling the whole volume, this prefactor scales with $L^{4}$ instead of $L$ as for a single photon, cf. [25].

\section{ACKNOWLEDGMENTS}

G. Torgrimsson is supported by the Alexander von Humboldt foundation.

\section{APPENDIX A: WORLD-LINE FORMALISM}

Here in the Appendix, we use for convenience units with $m=1$ and absorb the charge into the definition of the field strength, i.e. $q E \rightarrow E$.

Our starting point is again the worldline representation of the effective action. The spin factor can either be expressed in terms of a path-ordered exponential as in Sec. IV or as a path integral over an anticommuting Grassmann variable, $\psi_{\mu}(\tau)$, with antisymmetric boundary conditions, $\psi(1)=$ $-\psi(0)$,

$$
\begin{aligned}
\Gamma= & 2 \int_{0}^{\infty} \frac{\mathrm{d} T}{T} e^{-i \frac{T}{2}} \oint \mathcal{D} x \int \frac{\mathcal{D} \psi}{4} \\
& \times \exp \left(-i \int_{0}^{1} \mathrm{~d} \tau \frac{\dot{x}^{2}}{2 T}+A \dot{x}-\frac{i}{2} \psi \dot{\psi}-\frac{i}{2} \psi T F \psi\right),
\end{aligned}
$$

where $F_{\mu \nu}=\partial_{\mu} A_{\nu}-\partial_{\nu} A_{\mu}$. In this appendix we consider the superposition of a strong, constant field, $E$, and a plane wave with an arbitrary field shape, given by the potential $A_{0}=0$ and $\boldsymbol{A}(t, \boldsymbol{r})=E t \boldsymbol{e}_{z}+\varepsilon E \boldsymbol{e}_{P} \eta(n x)$, where the wave and polarization vectors satisfy $n_{\mu}=(1, \boldsymbol{n}), \boldsymbol{n}^{2}=1$, $\boldsymbol{e}_{P}^{2}=1, \boldsymbol{n} \cdot \boldsymbol{e}_{P}=0, n x=t+\boldsymbol{n} \cdot \boldsymbol{r}$, and where $f(n x)=$ $\eta^{\prime}(n x)$ is, at this point, an arbitrary function; $\eta(n x)=$ $\sin (\Omega n x) / \Omega$ gives the field considered in the main text. With $\varepsilon \ll 1$, we expand $\Gamma=\sum_{N=0}^{\infty} \varepsilon^{N} \Gamma_{N}$ and express the weak field in terms of its Fourier transform $\tilde{f}(\omega)$. The center of mass part of the worldline path integral gives an "energy conserving" delta function

$\int \mathrm{d}^{4} x_{\mathrm{cm}} \exp \left\{-i \sum_{i=1}^{N} k_{i} x_{\mathrm{cm}}\right\}=V_{3} 2 \pi \delta\left(\sum_{i=1}^{N} \omega_{i}\right)$,

where $k_{i, \mu}=\omega_{i} n_{\mu}$ and $\omega_{i}$ are the Fourier frequencies corresponding to the $N$ factors of $\tilde{f}$. The rest of the path integral is Gaussian and can be performed as in $[26,27,40]$ for $N$-photon amplitudes in constant fields. This involves the worldline Green's functions $\mathcal{G}_{B}$ and $\mathcal{G}_{F}$ for the $x$ and $\psi$ path integrals, respectively. For the exponential part of the probability we only need $\mathcal{G}_{B}$, which is given by

$$
\begin{aligned}
\mathcal{G}_{\mu \nu}^{B}(\tau)= & -\frac{i}{2 E} s\left(2\left[|\tau|-\tau^{2}\right]-\frac{1}{3}\right) g_{\mu \nu}^{\perp} \\
& -\frac{i}{2 E}\left(\frac{\cos [s(1-2|\tau|)]}{\sin s}-\frac{1}{s}\right) g_{\mu \nu}^{\|} \\
& +\frac{\epsilon(\tau)}{2 E}\left(\frac{\sin [s(1-2|\tau|)]}{\sin s}-(1-2|\tau|)\right) \hat{F}_{\mu \nu},
\end{aligned}
$$

where $s=i E T / 2$ and where the vector structure is determined by the direction of the strong field, i.e. $g_{\mu \nu}^{\|}=$ $\delta_{\mu}^{0} \delta_{\nu}^{0}-\delta_{\mu}^{3} \delta_{\nu}^{3}, g_{\mu \nu}^{\perp}=-\delta_{\mu}^{1} \delta_{\nu}^{1}-\delta_{\mu}^{2} \delta_{\nu}^{2}$, and $\hat{F}_{\mu \nu}=\delta_{\mu}^{0} \delta_{\nu}^{3}-\delta_{\mu}^{3} \delta_{\nu}^{0}$. This Green's function is the Minkowski version of the corresponding Euclidean Green's function, which can be found in $[26,40]$. In terms of this Green's function, we find that the dominant contribution to the $N$ th order is given by

$$
\begin{aligned}
\varepsilon^{N} P_{N}= & \operatorname{Im} \int \prod_{i=1}^{N} \mathrm{~d} \omega_{i} \tilde{f}\left(\omega_{i}\right) \delta\left[\sum_{i=1}^{N} \omega_{i}\right] \int_{0}^{\infty} \mathrm{d} s \int_{0}^{1} \prod_{i=1}^{N} \mathrm{~d} \tau_{i} \\
& \ldots \exp \left(-\frac{s}{E}-\frac{i}{2} \sum_{i, j=1}^{N} k_{i}\left[\mathcal{G}_{B}\left(\tau_{i}-\tau_{j}\right)-\mathcal{G}_{B}(0)\right] k_{j}\right),
\end{aligned}
$$


where the ellipses stand for subleading prefactor terms. The last term in (A3) does not contribute, because all $k_{i}$ are parallel and $k_{i} \hat{F} k_{j}=0$. The two terms proportional to $g^{\perp}$ and $g^{\|}$both lead to terms proportional to $n_{\perp}^{2} \omega_{i} \omega_{j}=$ $-k_{i} g^{\perp} k_{j}$, so, $n_{\perp} \omega_{i}$ gives an effective Fourier frequency. Since $\left|n_{\perp} \omega_{i}\right| \leq\left|\omega_{i}\right|$, the exponential is therefore maximized by plane waves traveling perpendicular to the strong field, i.e. for $n_{z}=0$. It follows from the delta function (A2) that we necessarily have both positive and negative frequencies. We label the frequencies such that $\omega_{i}>0$ for $i=1, \ldots, J$ and $\omega_{i}<0$ for $i=J+1, \ldots, N$. Consider each term in the sum in the exponent of (A4) separately. The term proportional to $\omega_{i} \omega_{j}$ is maximized by $\left|\tau_{i}-\tau_{j}\right|=0,1$ for $\omega_{i} \omega_{j}>0$ and by $\left|\tau_{i}-\tau_{j}\right|=1 / 2$ for $\omega_{i} \omega_{j}<0$. Similar to the saddle point method, we obtain the dominant exponential contribution by substituting these "maximizing" values of $\tau_{i}$ into (A4). This gives the following exponential for the $s$-integral:

$$
\exp \left\{-\frac{2 m_{\perp}^{2}}{E}\left(\frac{s}{2}-\Sigma^{2} \tan \frac{s}{2}\right)\right\}
$$

where we have defined

$$
\Sigma:=\frac{p_{\perp}}{m_{\perp}} \quad p_{\perp}:=\frac{1}{2} \sum_{i=1}^{J} k_{i \perp} \quad m_{\perp}:=\sqrt{1+p_{\perp}^{2}} .
$$

With the saddle point given by $s=2 \arccos \Sigma$, we find the general result

$$
\begin{aligned}
\varepsilon^{N} P_{N} \sim & \int \prod_{i=1}^{N} \mathrm{~d} \omega_{i} \tilde{f}\left(\omega_{i}\right) \delta\left(\sum_{i=1}^{N} \omega_{i}\right) \ldots \\
& \times \exp \left\{-\frac{2 m_{\perp}^{2}}{E}\left(\arccos \Sigma-\Sigma \sqrt{1-\Sigma^{2}}\right)\right\} \\
= & \int \prod_{i=1}^{N} \mathrm{~d} \omega_{i} \tilde{f}\left(\omega_{i}\right) \delta\left(\sum_{i=1}^{N} \omega_{i}\right) \ldots \\
& \times \exp \left\{-\frac{2}{E}\left(-p_{\perp}+m_{\perp}^{2} \arctan \frac{1}{p_{\perp}}\right)\right\} .
\end{aligned}
$$

With only one photon, $N=2$, we have $p_{\perp}=k_{\perp} / 2$, and the exponential in (A7) reduces to that in Eq. (5) in [25], as expected. Note that the exponential in (A7) has the same functional dependence of $\Sigma$ as for the longitudinal, purely time-dependent fields we considered in [14], see Eq. (3.4) in [14]. Comparing $\Sigma$ in (A6) with the corresponding quantity in Eq. (3.3) in [14], we see that the main difference in going from the purely time-dependent fields in [14] to the plane waves considered here is the appearance of a heavy effective mass, i.e. $m \rightarrow m_{\perp}>m$ (c.f. [41]), due to the spatial components of the wave vector. This means that plane waves will in general lead to less exponential enhancement than a purely time-dependent weak field.

\section{APPENDIX B: DOMINANT ORDER}

In this appendix we will present two methods for obtaining estimates of the dominant order, $N_{*}$, in the instanton formalism. These methods allow us to confirm the dominant order found using the approach in Appendix A.

Our starting point is the worldline representation of the effective action (A1). In the first method we focus on the scalar part of (A1), i.e. the part without Grassmann variables or Dirac matrices; we will show below using the second method that the spin factor does not significantly affect $N_{*}$. Let $a_{\mu} \propto \varepsilon$ be the weak field. For the fields we focus on in this paper, $a_{\mu}$ is a plane wave, but the methods we present here work also for more general field shapes. We expand the exponent in the weak field,

$$
\exp \left\{-i \int_{0}^{1} a \dot{x}\right\}=\sum_{N=0}^{\infty} \frac{1}{N !}\left(-i \int_{0}^{1} a \dot{x}\right)^{N}
$$
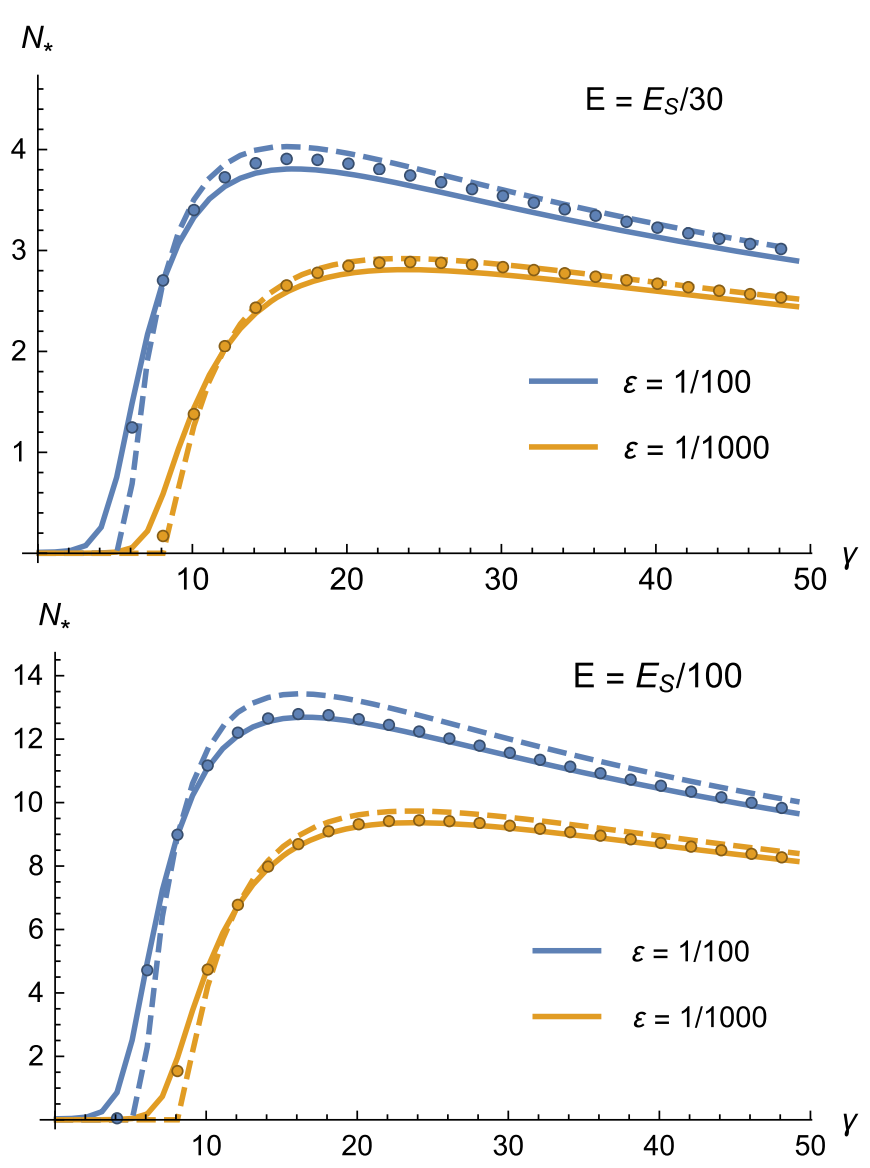

FIG. 5. These plots show our three estimates of the dominant order for perpendicular polarization. The dashed lines are obtained from Eq. (11), the solid lines show (B2), and the circles show (B4), in which $P_{e^{+} e^{-}}$is the total probability for spinor QED, including the prefactor. The third approach gives negative $N_{*}$ below the threshold, but that is just another sign (c.f. Fig. 4) that the instanton prediction of the prefactor breaks down in that regime [42]. 
and obtain an estimate of the dominant order from the "saddle point" of the sum over $N$. Assuming that the dominant order is "large", we use Stirling's approximation $\ln N ! \approx N(\ln N-1)$ and find

$$
N_{*}=-i \int_{0}^{1} a \dot{x}
$$

At this order we recover the original exponent, i.e.

$$
\frac{1}{N_{*} !}\left(-i \int_{0}^{1} a \dot{x}\right)^{N_{*}} \approx \exp \left\{-i \int_{0}^{1} a \dot{x}\right\}
$$

The instantons and the resulting exponential part of the probability will therefore be exactly the same as before, i.e. as without the additional steps (B1) to (B3). The point is that substituting the instantons into (B2) gives us a simple estimate of the dominant order in the instanton formalism. Note that, while we assume that $a_{\mu}$ is weak, the integral of $a_{\mu}$ in (B2) gives $N_{*}$, which is supposed to be large. So, in (B1) we expand the exponent in a parameter which is actually large. That is of course not a problem as it only means that we have to sum up many terms (the Taylor series for the exponential has an infinite radius of convergence). In fact, we want this expansion parameter to be large because in regimes where it is small, the dominant contribution comes from $N=0$ and then there is no significant enhancement of the probability. As shown in Fig. 5, the instanton estimate (B2) agrees with the previous estimate based on (11). So, (B2) seems to give a good estimate of the dominant order in dynamical assistance.
A more direct way of estimating the dominant order is to calculate the logarithmic derivative of the probability with respect to $\varepsilon$, i.e.,

$$
N_{*}=\frac{\mathrm{d} \log P_{e^{+} e^{-}}}{\mathrm{d} \log \varepsilon} .
$$

This expression is motivated by the fact that in a regime where $P_{e^{+} e^{-}} \sim \varepsilon^{N_{0}}$, (B4) gives $N_{*}=N_{0}$. In Fig. 5 we evaluate (B4) within the instanton formalism and show that (B4) agrees quite well with the simpler estimate of (B2). One advantage of (B4) is that it is general and does not depend on how we calculate $P_{e^{+}} e^{-}$. For example, for purely time dependent fields, like the ones we studied in [14], one can obtain the exact probability by solving the Riccati equation numerically, and then (B4) gives an exact description of how the probability depends on $\varepsilon$.

All the plots in Fig. 5 show qualitatively the same behavior as a function of $\gamma$ : below the threshold $N_{*} \approx 0$ (for $\varepsilon$ sufficiently small compared to $E$ ) [42], where the probability is given by Schwinger's constant field result. After the threshold, $N_{*}$ quickly reaches a maximum and then slowly decreases as $\gamma \rightarrow \infty$. So, pair creation actually becomes less "multiphoton" as $\gamma$ increases beyond the maximum. As Fig. 5 shows, the maximum dominant order can be quite large. However, it is not large for all relevant parameters. For e.g. $E=1 / 30$ and $\varepsilon=1 / 100$, the maximum dominant order is only $N_{*} \sim 4$, which suggests that it might be feasible in this case to actually calculate also the preexponential contributions to all important orders.
[1] E. Schrödinger, The proper vibrations of the expanding Universe, Physica (Utrecht) 6, 899 (1939).

[2] S. W. Hawking, Black hole explosions, Nature (London) 248, 30 (1974).

[3] S. W. Hawking, Particle creation by black holes, Commun. Math. Phys. 43, 199 (1975).

[4] F. Sauter, Über das Verhalten eines Elektrons im homogenen elektrischen Feld nach der relativistischen Theorie Diracs, Z. Phys. 69, 742 (1931).

[5] F. Sauter, Zum Kleinschen Paradoxon, Z. Phys. 73, 547 (1932).

[6] W. Heisenberg and H. Euler, Folgerungen aus der Diracschen Theorie des Positrons, Z. Phys. 98, 714 (1936).

[7] J. S. Schwinger, On gauge invariance and vacuum polarization, Phys. Rev. 82, 664 (1951).

[8] R. Schützhold, H. Gies, and G. Dunne, Dynamically Assisted Schwinger Mechanism, Phys. Rev. Lett. 101, 130404 (2008).

[9] M. Orthaber, F. Hebenstreit, and R. Alkofer, Momentum spectra for dynamically assisted Schwinger pair production, Phys. Lett. B 698, 80 (2011).
[10] A. Otto, D. Seipt, D. Blaschke, B. Kämpfer, and S. A. Smolyansky, Lifting shell structures in the dynamically assisted Schwinger effect in periodic fields, Phys. Lett. B 740, 335 (2015).

[11] A. Otto, D. Seipt, D. Blaschke, S. A. Smolyansky, and B. Kämpfer, Dynamical Schwinger process in a bifrequent electric field of finite duration: survey on amplification, Phys. Rev. D 91, 105018 (2015).

[12] M. F. Linder, C. Schneider, J. Sicking, N. Szpak, and R. Schützhold, Pulse shape dependence in the dynamically assisted Sauter-Schwinger effect, Phys. Rev. D 92, 085009 (2015).

[13] C. Schneider and R. Schützhold, Dynamically assisted Sauter-Schwinger effect in inhomogeneous electric fields, J. High Energy Phys. 02 (2016) 164.

[14] G. Torgrimsson, C. Schneider, J. Oertel, and R. Schützhold, Dynamically assisted Sauter-Schwinger effect-nonperturbative versus perturbative aspects, J. High Energy Phys. 06 (2017) 043.

[15] As an exception, it was shown in [12] that adding a standing (plane) wave $\propto \cos (k x) \cos (\omega t)$ to a constant field $E$ yields 
the same enhancement exponent as the corresponding purely time-dependent field. Furthermore, in [13] a spatially varying strong field plus a time-dependent weaker field is considered, which leads to a nontrivial interplay between the spatial and temporal dependences. In this context, we would also like to mention a recent paper [16] which studies pair production in the collision of two plane waves.

[16] I. A. Aleksandrov, G. Plunien, and V. M. Shabaev, Momentum distribution of particles created in space-time-dependent colliding laser pulses, Phys. Rev. D 96, 076006 (2017).

[17] I. A. Batalin and E. S. Fradkin, Quantum electrodynamics in external fields. I, Teor. Mat. Fiz. 5, 190 (1970); [Theor. Math. Phys. 5, 1080 (1970)].

[18] N. B. Narozhnyi and A. I. Nikishov, Teor. Mat. Fiz. 26, 16 (1976).[Solutions of the Klein-Gordon and Dirac equations for a particle in a constant electric field and a plane electromagnetic wave propagating along the field, Theor. Math. Phys. 26, 9 (1976)].

[19] E. S. Fradkin, D. M. Gitman, and S. M. Shvartsman, Quantum Electrodynamics with Unstable Vacuum (Springer series in nuclear and particle physics, Berlin, 1991).

[20] D. M. Gitman and S. I. Zlatev, Spin factor in path integral representation for Dirac propagator in external fields, Phys. Rev. D 55, 7701 (1997).

[21] T. N. Tomaras, N. C. Tsamis, and R. P. Woodard, Back reaction in light cone QED, Phys. Rev. D 62, 125005 (2000).

[22] T. N. Tomaras, N. C. Tsamis, and R. P. Woodard, Pair creation and axial anomaly in light cone QED(2), J. High Energy Phys. 11 (2001) 008.

[23] A. Ilderton, Localisation in worldline pair production and lightfront zero-modes, J. High Energy Phys. 09 (2014) 166.

[24] A. Ilderton, G. Torgrimsson, and J. Wårdh, Nonperturbative pair production in interpolating fields, Phys. Rev. D 92 , 065001 (2015).

[25] G. V. Dunne, H. Gies, and R. Schützhold, Catalysis of Schwinger vacuum pair production, Phys. Rev. D 80, 111301 (2009).

[26] C. Schubert, Perturbative quantum field theory in the string inspired formalism, Phys. Rep. 355, 73 (2001).

[27] C. Schubert, Lectures on the Worldline Formalism, School on Spinning Particles in Quantum Field Theory: Worldline Formalism, Higher Spins, and Conformal Geometry.
[28] For example, in the limit $\Omega \rightarrow 0$ we have a constant field with a field strength $E(1+\varepsilon)$, and by expanding Schwinger's result one finds that all orders in $\varepsilon$ are nonzero.

[29] I. K. Affleck, O. Alvarez, and N. S. Manton, Pair production at strong coupling in weak external fields, Nucl. Phys. B197, 509519 (1982).

[30] G. V. Dunne and C. Schubert, Worldline instantons and pair production in inhomogenous fields, Phys. Rev. D 72 , 105004 (2005).

[31] G. V. Dunne, Q. Wang, H. Gies, and C. Schubert, Worldline instantons and the fluctuation prefactor, Phys. Rev. D 73, 065028 (2006).

[32] G. V. Dunne and Q. Wang, Multidimensional worldline instantons, Phys. Rev. D 74, 065015 (2006).

[33] C. Schneider and R. Schützhold, Prefactor in the dynamically assisted Sauter-Schwinger effect, Phys. Rev. D 94, 085015 (2016).

[34] C. Schneider et al., Discrete worldline instantons (in preparation).

[35] W. Rheinboldt, Numerical continuation methods: A perspective, J. Comput. Appl. Math. 124, 229 (2000).

[36] O. Gould and A. Rajantie, Thermal Schwinger pair production at arbitrary coupling, Phys. Rev. D 96, 076002 (2017).

[37] L. D. Faddeev and V. N. Popov, Feynman diagrams for the Yang-Mills field, Phys. Lett. 25B, 2930 (1967).

[38] J. Gordon and G. W. Semenoff, World-line instantons and the Schwinger effect as a Wentzel-Kramers-Brillouin exact path integral, J. Math. Phys. (N.Y.) 56, 022111 (2015).

[39] European XFEL, www.xfel.eu.

[40] C. Schubert, Vacuum polarization tensors in constant electromagnetic fields. Part 1., Nucl. Phys. B585, 407 (2000).

[41] G. Torgrimsson, J. Oertel, and R. Schützhold, Doubly assisted Sauter-Schwinger effect, Phys. Rev. D 94, 065035 (2016).

[42] If one estimates the dominant order by calculating $P_{e^{+} e^{-}}$in (B4) using the instanton formalism, then one might obtain incorrect results below the threshold, e.g. $N_{*} \approx-1$. This is because, in that regime, terms in the exponent that were assumed to be large are actually not. One can understand this in the limit $\gamma \rightarrow 0$ by expanding the (locally) constant field result in $\varepsilon$. 